\title{
Reconfigurable Self-Assembly of Mesoscale Optical Components at a Liquid-Liquid Interface
}

\section{Citation}

Tang, Sindy K.Y., Ratmir Derda, Aaron D. Mazzeo, and George M. Whitesides. 2011.

"Reconfigurable Self-Assembly of Mesoscale Optical Components at a Liquid-Liquid Interface." Advanced Materials 23, no. 21: 2413-2418.

\section{Published Version}

doi:10.1002/adma.201100067

\section{Permanent link}

http://nrs.harvard.edu/urn-3:HUL.InstRepos:12967835

\section{Terms of Use}

This article was downloaded from Harvard University's DASH repository, and is made available under the terms and conditions applicable to Open Access Policy Articles, as set forth at http:// nrs.harvard.edu/urn-3:HUL.InstRepos:dash.current.terms-of-use\#OAP

\section{Share Your Story}

The Harvard community has made this article openly available.

Please share how this access benefits you. Submit a story.

\section{Accessibility}




\title{
Reconfigurable Self-Assembly of Mesoscale Optical Components at a Liquid-Liquid Interface
}

\author{
Sindy K.Y. Tang, Ratmir Derda, Aaron D. Mazzeo, and George M. Whitesides* \\ Department of Chemistry and Chemical Biology, 12 Oxford Street, Harvard University \\ Cambridge, MA 02138 \\ *gwhitesides@gmwgroup.harvard.edu
}

\section{Keywords:}

Self-Assembly, Functional Coatings, Surface Modification, Diffraction Gratings 
We describe a method that uses magnetic fields to template the self-assembly and reconfiguration of 2-D optical components with engineered surface wettabilities at a liquid-liquid interface. The optical components are mesoscale tiles—diffraction gratings—each fabricated to contain a magnetic strip. Application of a magnetic field to the tiles—suspended at the interface between two immiscible liquids—assembles them into an array of gratings. The orientations of the tiles, and the resulting optical effects, are reconfigurable by a change in the magnetic field. It is possible to preserve the assembled patterns, if desired, by transferring them onto solid substrates. This procedure can be useful for generating coatings or films with interesting optical effects, and for visualizing magnetic fields.

Self-assembly methods allow the distribution and alignment of small, preformed objects into desired patterns. ${ }^{[1-4]}$ It is a potential alternative to conventional top-down techniques (e.g. photolithography) and serial processing steps (e.g. pick-and-place robotics) for scalable and lowcost fabrication of a wide range of structures with length scales from nanometers (e.g. selfassembly of diblock copolymers, ${ }^{[5-8]}$ templated self-assembly using immunoglobulins, ${ }^{[9]}$ DNA and virus ${ }^{[10-14]}$ ) to centimeters. ${ }^{[1,15]}$ Self-assembly can generate materials with unique and useful optical properties: for example, 3D-assembly of colloids can form photonic crystals, ${ }^{[16-17]}$ suspensions of silver nanoparticles ${ }^{[18]}$ and gold-coated silicon hexagons ${ }^{[19]}$ provide the reflective element for liquid mirrors, selective wetting of polymers on long hydrophobic strips forms optical waveguides. ${ }^{[20]}$ The organization of mesoscale $(\mathrm{mm}-\mathrm{cm})$ optical modules is less explored than that of nano-scale structures. Mesoscale structures offer the potential to generate regular arrays of functional optical elements over large areas. Some functions (e.g. diffraction, lensing) cannot be obtained easily (or at all) from the direct assembly of nanostructures. 
We and others have previously demonstrated the self-assembly of mesoscale plates into various arrays and porous structures using lateral capillary interactions between menisci at the edges of the plates. ${ }^{[21-31]}$ Most of these assemblies were static: once assembled, it was difficult to reconfigure the assembled pattern. There are few strategies that allow real-time reconfiguration of self-assembled mesoscale structures without requiring a change in the design and fabrication of individual components. Mao et. al. showed the reconfiguration of assembled structures by changing the center of mass of the plates suspended between two immiscible liquids: the assembled pattern changed from one where positive menisci dominated their self-assembly, to one where negative menisci dominated. ${ }^{[28]}$ This method was limited, however: there were only two possible configurations; it also required a change of the liquids used.

This paper describes a simple approach to the dynamically reconfigurable self-assembly of more than 300 tiles of millimeter-scale diffraction gratings over areas greater than $10 \mathrm{~cm}^{2}$. Changing the magnetic fields changes the patterns of the assembled structure and generates a range of optical effects. Anisotropic modification of the wettability of the top and bottom surfaces of the tiles locks them in the $x-y$ plane at the interface between a hydrophobic liquid and a hydrophilic liquid with uniform top-down orientations. We did not modify the wettability of the side edges of the tiles using established methods. ${ }^{[21-31]}$ Had we done so, the assembled structure could have been locked into a particular configuration by capillary interactions, or caused to have attractive or repulsive interactions. The aggregates of tiles with unmodified edges disassembled upon the removal of the magnetic field (by agitation, for example), and reconfigured into new structures upon the application of different patterns of magnetic fields. 
This approach to the generation of reconfigurable optical films has five characteristics: (i) the application of a magnetic field is required to assemble and orient the tiles. The orientation of the tiles in the assembled pattern follows the lines of the magnetic field. (ii) Because there is no solid friction at the liquid-liquid interface, the optical elements are not subject to stiction or wear. (iii) The assembled pattern could be reconfigured as many times as desired by a change in the magnetic field. (iv) This method can, in principle, be used to organize and register large number of micro- to millimeter-scale components on surfaces (flat or curved). It is also interesting for its ability to generate colorful and changeable optical effects, and to visualize magnetic field lines. (v) Assembled pattern can be retained permanently by transferring the tiles onto a solid substrate; it makes this approach useful for the fabrication of optically functional coatings or films.

Assembly of gratings using templating by a magnetic field: We fabricated tiles (squares with edges of $2 \mathrm{~mm}$, and circles with diameters of $2 \mathrm{~mm}$ ) of reflective diffraction elements with the top surface made hydrophilic, and the opposite face made hydrophobic (Figure 1, see Experimental section for details). We dispersed these tiles in a Petri dish containing PFMD and water (Figure 2a). The density of the polyester tiles was about $1.4 \mathrm{~g} \mathrm{~cm}^{-3}$ : that is, intermediate between that of PFMD $\left(\rho \sim 2 \mathrm{~g} \mathrm{~cm}^{-3}\right)$ and water $\left(\rho \sim 1 \mathrm{~g} \mathrm{~cm}^{-3}\right)$. The tiles self-assembled at the interface of the two liquids with an orientation driven by the pattern of hydrophilic and hydrophobic surface modification on the two faces of the tiles. After shaking or stirring the suspension for a few seconds, $99 \%$ tiles settled into the correct up-down orientation, in which the hydrophilic side faced the water phase, and the hydrophobic side faced the PFMD. 
Additional shaking did not flip upside-down tiles into the proper orientation. More vigorous agitation could help, but excessive shaking might cause the tiles to overlap.

We applied a magnetic field to the system of tiles at the liquid-liquid interface using a rectangular $\mathrm{NdFeB}$ bar magnet $(4 \mathrm{~cm} \times 4 \mathrm{~cm} \times 1 \mathrm{~cm}$, in the $x, y$, and $z$ directions), placed a few centimeters beneath the liquid-liquid interface. Upon application of this magnetic field, the tiles self-assembled into a structure such that the strips of iron powder on their bottom side aligned with the direction of the magnetic field (Figure 2c-e). The tiles, thus, mapped the lines of the field produced by the magnets. The time necessary to assemble the tiles into the final pattern increased as the strength of the magnetic field decreased (Figure 2b). For field strengths $<10$ mT (measured by a Hall-effect gauss meter, Sypris Model 6010), the tiles did not assemble into a close-packed structure. For field strengths $>120 \mathrm{mT}$, the tiles were no longer fixed at the interface between water and PFMD: they were pulled through the interface to the bottom of Petri dish closest to the magnets. For the experiments that involved orienting the tiles at the interface, applied field strengths were between 80 and $100 \mathrm{mT}$.

After some agitation (e.g. by gentle tapping on the Petri dish for 5 seconds), the tiles assembled into a structure that was $80-90 \%$ close-packed. This number was estimated from the ratio of (area of a perfect close-packed structure that contains the same number of components)/(area of the assembled structure). The theoretical maximum packing density of tiles is $90.67 \%$ in a close-packed hexagonal lattice composed of circular tiles, while the theoretical maximum packing density is $100 \%$ in a square lattice composed of square tiles. The patterns of colors reflected from square tiles assembled in a square lattice, therefore, appeared 
more uniform than the patterns reflected from assemblies of circular tiles (compare Figure 2e, bottom, and Figure 6b).

Figure 3 shows the assembled tiles of gratings upon the application of a magnetic field. The field lines were parallel in the $x$-direction over the whole area of the Petri dish. The long axis of the grooves on the gratings was in the $y$-direction. We varied the angle of observation in the $x$-z plane, and examined the colors reflected. Due to the diffraction of light by the grating elements, the colors observed at different angles follows that given by the grating equation (Eq. $1):{ }^{[32]}$

$$
d\left(\sin \theta_{r}-\sin \theta_{i}\right)=\mathrm{m} \lambda
$$

where $d(=1 \mu \mathrm{m})$ is the spacing between the grooves in the grating, $\theta_{i}\left(\sim 30^{\circ}\right)$ is the angle of the light source, $\theta_{r}$ is the angle of reflection of diffraction order $\mathrm{m}$, and $\lambda$ is the wavelength of light ( $\lambda \sim 400 \mathrm{~nm}-700 \mathrm{~nm}$ for the visible wavelengths). At $\theta_{r} \sim 30^{\circ}$, diffraction of order $\mathrm{m}=0$ occurred; no dispersion of color could be observed (Figures 3b-ii, 3c-ii). When the observation angle was at $\sim 5^{\circ}$ (Figure 3c-iv), a large range of colors spanning from blue to red could be imaged with our camera. These colors originated from the diffraction of visible wavelengths with order $\mathrm{m}=-1\left(\theta_{r, m=-1, \lambda=400 \mathrm{~nm}} \sim 5^{\circ}\right.$, and $\left.\theta_{r, m=-1, \lambda=700 \mathrm{~nm}}=-11^{\circ}\right)$ (Figure 3b-iv). ${ }^{[33]}$

Although optimizing order in the assemblies was not our highest priority, defects in the assembled structures were noticeable based on the profiles of the light reflected: (i) There were voids among the tiles. For square tiles, these voids came, at least in part, from differences in the dimensions of the manually-cut tiles. The magnetic strips were also not always centered (+/- 0.2 $\mathrm{mm}$ from the center of the tile). (ii) The colors and intensities of light reflected were not 
uniform. These non-uniformities arose from small tilts of the tiles at the liquid-liquid interface, probably due to small irregularities at the edges of the tiles formed in fabrication, and consequent irregularities in the forces produced by the meniscus. For circular tiles, the puncher we used to cut the tiles also caused the tile to curve. (iii) Tiles occasionally overlapped if agitation was too rigorous.

Many of these defects can be minimized by improving the fabrication of the tiles. The most difficult issue with fabrication here was the cutting of uniformly shaped gratings with no curl or deformities around the edges. These difficulties can, in part, be mitigated using existing methods in micro-fabrication.

Dynamic reconfiguration of the assembled pattern by a change in the applied magnetic field: Figures 4-5 show that the pattern of the assembled tiles depended on the pattern of the applied magnetic field. The tiles assembled into structures in which the magnetic strips under each tile were aligned with the component of the local magnetic field lines projected in the plane of the liquid-liquid interface. Since the gratings consisted of linear grooves, the diffraction of lightand therefore the distribution of reflected colors from the tiles—occurred at a relatively narrow range of angles, when the grooves on the grating are perpendicular to the direction of observation. When the applied magnetic field lines were not unidirectional (Figure 4), the orientations of the gratings on the tiles were not uniform throughout the whole assembly. Only a small fraction of tiles reflected bright colors under these magnetic field patterns. 
To obtain reflection of colors from a large range of angles, other designs of diffraction gratings can be used (e.g. one that consists of a regular array of posts). Alternatively, the use of photonic crystals with complete band gaps ${ }^{[34]}$ allows the reflection of light in all directions at fixed wavelengths. To achieve changeable optical effects by a rotation in magnetic fields, the tile elements should also display rotational asymmetry in its optical properties. So long as magnetically-responsive components can be incorporated into these optical elements, the same strategy can be used to assemble these elements using a magnetic field.

Transfer of grating tiles onto solid substrates: One disadvantage of the assembly of these tiles at a liquid-liquid interface is that it is difficult to preserve the patterns permanently, or to integrate them with other materials or devices. Here we demonstrate an approach that transfers the assembled pattern onto solid and flexible substrates.

We assembled the tiles at the interface of PFMD and an agarose solution (1 wt \%) at 60 ${ }^{\circ} \mathrm{C}$ (Figure 6). After the agarose cooled to $20{ }^{\circ} \mathrm{C}$ and gelled, the tiles were fixed on the surface of the gel. Upon draining of the PFMD, the bottom surface of the tiles became exposed. Curing PDMS on this surface, and subsequent melting the agarose and pouring it off, transferred the tiles onto PDMS. Figure 6e,f show that it was possible to bend this PDMS film or wrap it around a wrist. In principle, it is possible to suspend the tiles at the interface of PDMS and PFMD, or PDMS and water directly. Curing the PDMS after the tiles assembled under a magnetic field could generate a film of PDMS consisting of the assembled tiles. This strategy was not practical using the materials we had available because PDMS was more viscous than 
water, and the time for the tiles to assemble was $>10$ times longer than that needed for PFMD/water system.

Conclusions: We demonstrated a new method for the assembly and registration of optical components with small footprints using surface forces and magnetic forces. To obtain different optical responses (reflected colors here) from these components, we applied the magnetic fields in different orientations relative to the light source. We have shown the manipulation of the orientation of the tiles in the plane of the liquid-liquid interface ( $x$-y plane) only. It should also be interesting to control the tilting angle of these tiles (in the $x$-z, or $y$-z planes): the tilt of the grating element determines the color reflected at fixed angles of light source and observation. If the tilting of individual elements can be addressed separately, our system can be applied towards constructing a reflection-based color display.

We have also demonstrated a way to transfer assembled materials from liquid interfaces onto solid substrates. Our method can, therefore, be compatible with micro- or nano-fabrication procedures where the components were formed in a liquid. Nano-scale optical structures (e.g. metamaterials with negative index of refraction) fabricated using nanoskiving, ${ }^{[35-36]}$ for example, are suspended at the interface of water and air after being sliced with a microtome. The footprint of these elements is small (a few hundreds of $\mu \mathrm{m}^{2}$ ). While this size is sufficient for characterization, it would be very difficult to fabricate these components in large areas necessary for applications in coatings, or films (e.g., construction of an invisibility cloak ${ }^{[37]}$ directly from micron-sized samples with negative index properties would be very challenging). If magnetic components can be incorporated into these elements, however, assembly of these elements into 
large areas should be possible using the procedure we have described. We have shown the assembly of hundreds of structures over areas of $\sim \mathrm{cm}^{2}$ using commercially-available permanent magnets; electromagnets can potentially generate fields over much larger areas than permanent magnets for the assembly of many elements over much larger areas $\left(\sim \mathrm{m}^{2}\right)$. We believe that our assembly method can bridge a gap between nano-fabrication (where sample sizes are usually small) and the generation of large surface-area materials for end use.

\section{Experimental}

Fabrication of tiles of gratings: We coated one side of a $100-\mu \mathrm{m}$ thick transmission-type polyester diffraction grating film (1000 grooves/mm, Edmund Optics) with $150 \mathrm{~nm}$ of platinum to make the grating reflective (Figure 1). Using Pt was advantageous because: (i) it was possible to form self-assembled monolayers (SAM) of thiols on them to modify the wettability of the surface; ${ }^{[38]}$ (ii) Pt adhered to the surface of polyester without the need of an adhesion layer; (iii) Among metals on which we could form self-assembled monolayers of thiols (Au, Ag, Pt, Pd), ${ }^{[38]}$ the reflectivity of light from Pt was relatively uniform in the visible wavelengths. Au has low reflectivity at wavelengths $<500 \mathrm{~nm}$ (and therefore the yellowish reflected color). Reflectivity from Ag degraded over time due to the formation of silver oxide.

To fabricate magnetic strips on the opposite face of the grating, we attached $0.3 \mathrm{~mm}$-wide strips of double-sided tape (indoor carpet tape, 3M), generated using a laser cutter. Pressing this surface against a plate covered with loose iron powders coated the strips of double-sided tape with a sufficient quantity of ferromagnetic powders to make them strongly responsive to 
magnetic fields. The incorporation of these magnetic strips allowed the position and orientation of the gratings to be manipulated using an external magnetic field.

Immersion of the samples in a solution of 3-mercapto-1-propanesulfonic acid (1 $\mathrm{mM}$ in ethanol, $5 \mathrm{~min}$ ) formed hydrophilic SAMs on Pt-coated side. The contact angles of water and perfluoro-1-methyldecalin (PFMD) on this surface were both $<10^{\circ}$. We used a pipette to apply amorphous fluoropolymer (Teflon AF 400S1-100-1, Du Pont) to the opposite surface; the fluoropolymer spread evenly and dried to form a continuous hydrophobic film. The contact angles of water and PFMD on this surface were $120^{\circ}$, and $<10^{\circ}$ respectively. These grating components therefore had a strong orientation preference when suspended and agitated at the interface between water and perfluorocarbon: they oriented spontaneously in such a way that the hydrophilic side, which contained the grating structure, was in contact with water, while the Teflon-coated side, which contained the magnetic strip, was in contact with the perfluorocarbon.

Tiles also spontaneously oriented themselves at the interface of water and some hydrocarbons (e.g. hexane) with the hydrophobic side facing the top hydrocarbon phase, and the hydrophilic side facing the bottom water phase. If this hexane-water system were to be used, the surface wettability of the tiles would have to be reversed so that the grating elements would face up. ${ }^{[39]}$ Organic solvents (e.g. dichloromethane) that are denser than water tend to dissolve these tiles; by working with a cross-linked polymer, it would be possible to make them much more solvent resistant. For all our self-assembly experiments, we used perfluorocarbons instead of hydrocarbons because perfluorocarbons are non-toxic and chemically inert.

We cut the grating into square shapes (2 mm x $2 \mathrm{~mm}$ ) using scissors, or into circular shapes (diameter $2 \mathrm{~mm}$ ) using a hole puncher (McMaster). We selected this size because tiles of 
larger footprint did not orient spontaneously at the liquid-liquid interface, and tiles of smaller size were challenging to fabricate manually.

\section{Acknowledgments}

We thank Dr. Claudiu Stan and Qimin Qiao for helpful discussions. This work is supported by the Defense Advanced Research Projects Agency (DARPA) Chemical Communications and Programmable Matter.

\section{References}

[1] M. Boncheva, D. A. Bruzewicz, G. M. Whitesides, Pure Appl. Chem. 2003, 75, 621.

[2] M. Boncheva, G. M. Whitesides, Mater. Res. Bull. 2005, 30, 736.

[3] G. M. Whitesides, M. Boncheva, Proc. Natl. Acad. Sci. U. S. A. 2002, 99, 4769.

[4] G. M. Whitesides, B. Grzybowski, Science. 2002, 295, 2418.

[5] K. C. Daoulas, M. Muller, M. P. Stoykovich, S. M. Park, Y. J. Papakonstantopoulos, J. J. de Pablo, P. F. Nealey, H. H. Solak, Phys. Rev. Lett. 2006, 96.

[6] S. A. Morin, Y. H. La, C. C. Liu, J. A. Streifer, R. J. Hamers, P. F. Nealey, S. Jin, Angew. Chem., Int. Ed. 2009, 48, 2135.

[7] M. P. Stoykovich, M. Muller, S. O. Kim, H. H. Solak, E. W. Edwards, J. J. de Pablo, P. F. Nealey, Science. 2005, 308, 1442.

[8] X. M. Yang, R. D. Peters, P. F. Nealey, H. H. Solak, F. Cerrina, Macromolecules. 2000, 33, 9575. 
[9] J. Yang, M. Mayer, J. K. Kriebel, P. Garstecki, G. M. Whitesides, Angew. Chem., Int. Ed. 2004, 43, 1555.

[10] R. L. Fan, A. L. Boyle, V. V. Cheong, S. L. Ng, B. P. Orner, Biochemistry. 2009, 48, 5623.

[11] S. Mann, Nat. Mater. 2009, 8, 781.

[12] W. M. Shih, C. X. Lin, Curr. Opin. Struct. Biol., 20, 276.

[13] M. Uchida, S. Kang, C. Reichhardt, K. Harlen, T. Douglas, Biochim. Biophys. Acta, Gen. Subj., 1800, 834.

[14] Y. Zhang, S. Raudah, H. Teo, G. W. S. Teo, R. L. Fan, X. M. Sun, B. P. Orner, J. Biol. Chem., 285, 12078.

[15] A. Terfort, N. Bowden, G. M. Whitesides, Nature. 1997, 386, 162.

[16] A. Imhof, D. J. Pine, Nature. 1997, 389, 948.

[17] J. Wijnhoven, W. L. Vos, Science. 1998, 281, 802.

[18] E. F. Borra, A. Ritcey, E. Artigau, Astrophys. J. 1999, 516, L115.

[19] M. A. Bucaro, P. R. Kolodner, J. A. Taylor, A. Sidorenko, J. Aizenberg, T. N. Krupenkin, Langmuir. 2009, 25, 3876.

[20] E. Kim, G. M. Whitesides, L. K. Lee, S. P. Smith, M. Prentiss, Adv. Mater. 1996, 8, 139.

[21] N. Bowden, F. Arias, T. Deng, G. M. Whitesides, Langmuir. 2001, 17, 1757.

[22] N. Bowden, I. S. Choi, B. A. Grzybowski, G. M. Whitesides, J. Am. Chem. Soc. 1999, 121, 5373.

[23] N. Bowden, S. R. J. Oliver, G. M. Whitesides, J. Phys. Chem. B. 2000, 104, 2714.

[24] N. Bowden, A. Terfort, J. Carbeck, G. M. Whitesides, Science. 1997, 276, 233.

[25] I. S. Choi, N. Bowden, G. M. Whitesides, J. Am. Chem. Soc. 1999, 121, 1754. 
[26] T. D. Clark, R. Ferrigno, J. Tien, K. E. Paul, G. M. Whitesides, J. Am. Chem. Soc. 2002, 124, 5419.

[27] W. T. S. Huck, J. Tien, G. M. Whitesides, J. Am. Chem. Soc. 1998, 120, 8267.

[28] C. D. Mao, V. R. Thalladi, D. B. Wolfe, S. Whitesides, G. M. Whitesides, J. Am. Chem. Soc. 2002, 124, 14508.

[29] S. R. J. Oliver, T. D. Clark, N. Bowden, G. M. Whitesides, J. Am. Chem. Soc. 2001, 123, 8119.

[30] D. B. Wolfe, A. Snead, C. Mao, N. B. Bowden, G. M. Whitesides, Langmuir. 2003, 19, 2206.

[31] H. K. Wu, N. Bowden, G. M. Whitesides, Appl. Phys. Lett. 1999, 75, 3222.

[32] E. Hecht, in Optics, Addison Wesley, San Francisco 2002, 10.

[33] We note that the calculated values of angles of diffraction were only approximate since the light source was not collimated; the incident light had a divergence angle of $\sim 5-10^{\circ}$. Using the same calculations, we estimated that diffraction of $400 \mathrm{~nm}$-light of order $\mathrm{m}=1$ occurred at $\theta_{r}$, $\mathrm{m}=1, \lambda=400 \mathrm{~nm} \sim 64^{\circ}$. Some trace of blue could be seen at $\theta_{r} \sim 60^{\circ}$ (Figures 3b-i, and 3c-i).

Diffraction of $400 \mathrm{~nm}$-light of order $\mathrm{m}=-1$ occurred at $\theta_{r} \sim 5^{\circ}$. Trace of blue could be seen at $\theta_{r}$, $\mathrm{m}=-1, \lambda=400 \mathrm{~nm} \sim 10^{\circ}$ (Figure 3b-iii, 3c-iii). The lens in our camera had an f-stop of 32, corresponding to an NA of $\sim 0.02$ and a viewing half-angle of $\sim 1^{\circ}$.

[34] J. D. Joannopoulos, R. D. Meade, J. N. Winn, Photonic Crystals: Molding the Flow of Light, Princeton University Press, New Jersey 1995.

[35] D. J. Lipomi, R. V. Martinez, R. M. Rioux, L. Cademartiri, W. F. Reus, G. M. Whitesides, ACS Appl. Mater. Interfaces. 2010, 2, 2503. 
[36] Q. B. Xu, R. M. Rioux, M. D. Dickey, G. M. Whitesides, Acc. Chem. Res. 2008, 41, 1566.

[37] T. Ergin, N. Stenger, P. Brenner, J. B. Pendry, M. Wegener, Science. 2010, 328, 337.

[38] J. C. Love, L. A. Estroff, J. K. Kriebel, R. G. Nuzzo, G. M. Whitesides, Chem. Rev. 2005, 105, 1103.

[39] Tiles (density $~ 1.4 \mathrm{~g} \mathrm{~cm}^{-3}$ ) were unstable at water-hexane interface; they sank irreversibly upon agitation. Salts (e.g. caesium chloride) could be added to water to increase its density for stable suspension of tiles. 
Figure 1. (a) Fabrication of grating tiles. We deposited $150 \mathrm{~nm}$-thick of platinum on a transmission-type polyester film of grating. We attached strips of double-sided tape to the bottom face of the gratings, and coated them with iron powder to make them responsive to magnetic fields. We rendered the Pt-coated side of the grating hydrophilic with 3-mercapto-1propanesulfonic acid, and the opposite side hydrophobic with Teflon AF. The tiles were then cut into square (length $=2 \mathrm{~mm}$ ) or circular shapes (diameter $=2 \mathrm{~mm}$ ) manually using scissors or hole punchers. (b) Scheme showing the top and bottom of the tile. (c) Optical micrograph of four tiles. The left shows the top face of the tiles, and the right shows the bottom face of the tiles. The white arrows indicate the direction of the grating grooves. The reflected colors from the top and bottom surfaces of the shown tiles differed because the tiles on the left were tilted slightly into the plane relative to the tiles on the right.

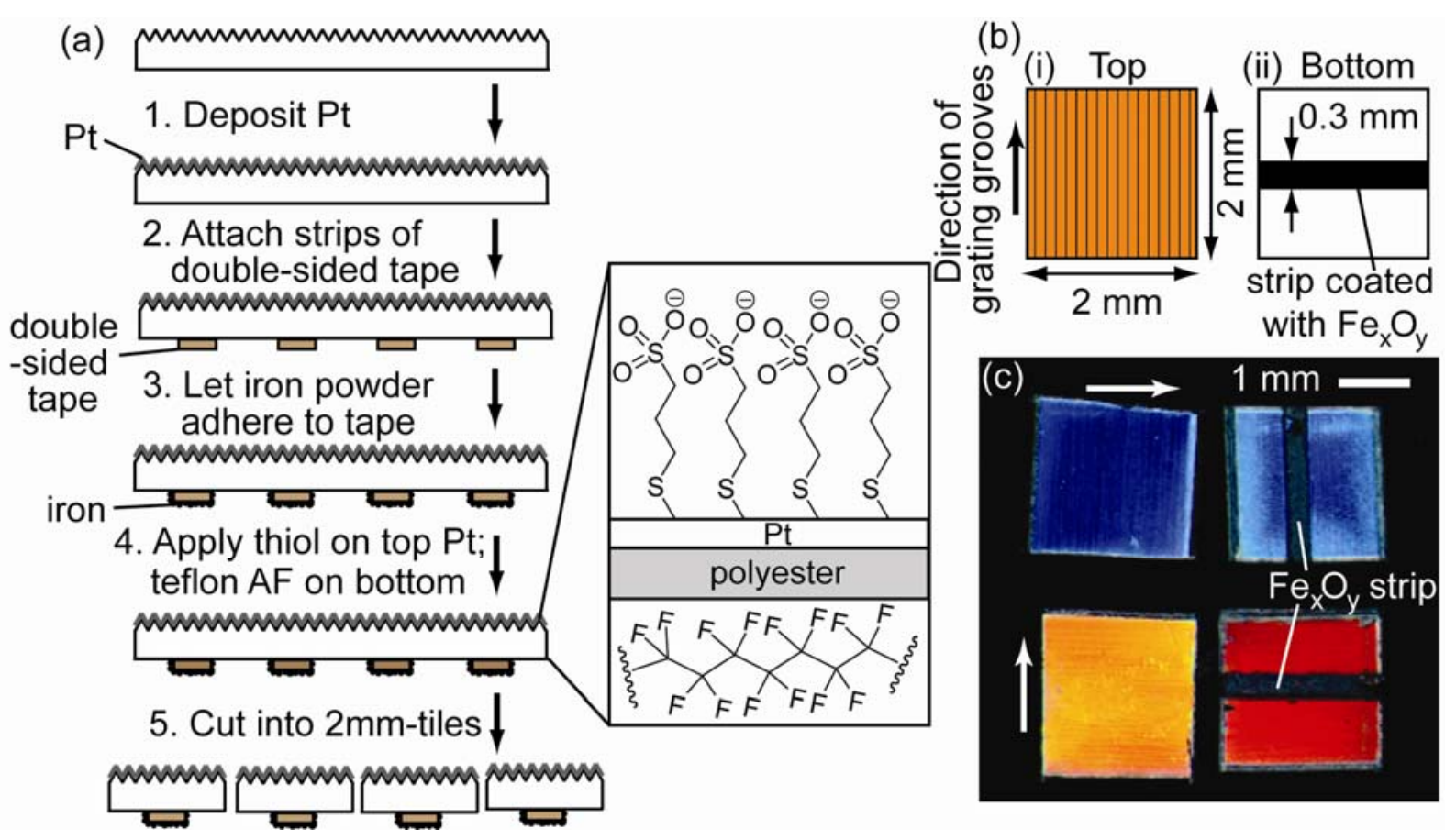


Figure 2. (a) Scheme of the positioning of the tiles at the water-PFMD interface. (b) The time needed to assemble the tiles into the final pattern as a function of the strength of magnetic field. (c) to (e): Schemes and photographs showing the orientation and alignment of the tiles with external magnetic field.
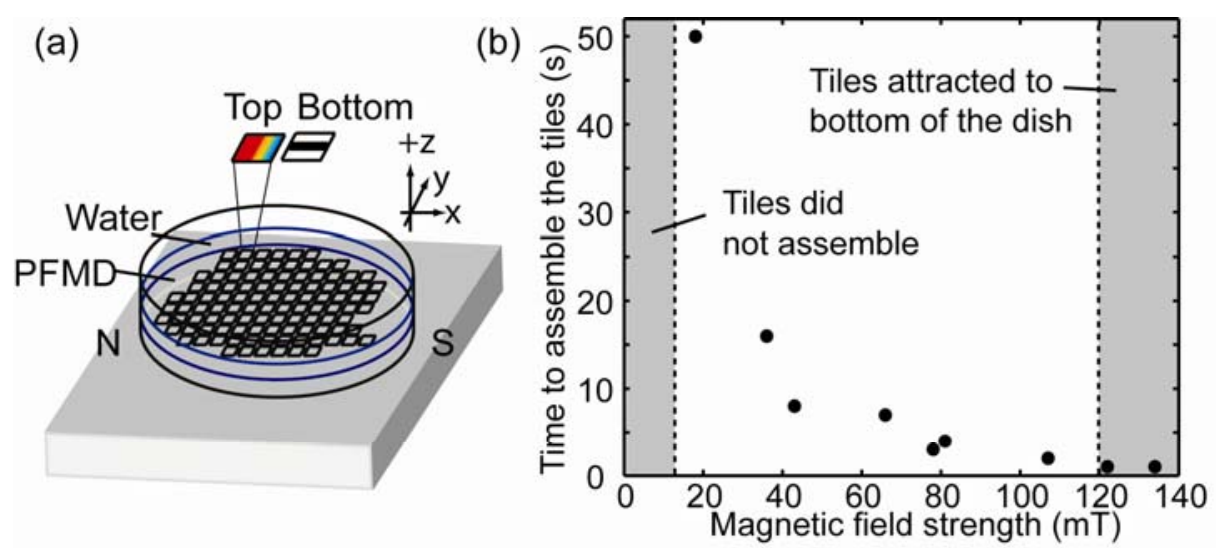

(c) Top View

(d) Bottom View

(e) Photograph
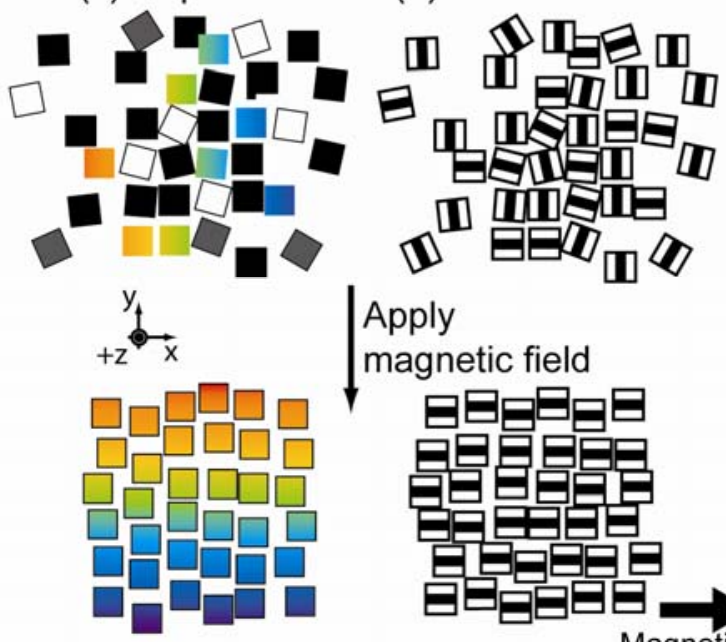

Apply magnetic field


Field 
Figure 3. (a) Scheme showing the conditions at which optical micrographs in (c) were obtained. The angle of the incident light $\left(\theta_{\mathrm{i}}\right)$ was fixed, and the angle of observation $\left(\theta_{\mathrm{r}}\right)$ varied from $\sim 5^{\circ}$ to $60^{\circ}$. A uniform magnetic field was applied in the $x$-direction. (b) shows a simplified scheme of the diffraction of visible light from a small area on the grating. The actual light source was not collimated. (i) to (iv) indicate the approximate angles of the camera in (c). (c) shows photographs of the assembled tiles imaged at different observation angles. The magnetic field was fixed in all cases. The length and width of the tiles were both $2 \mathrm{~mm}$.

(a) Broadband

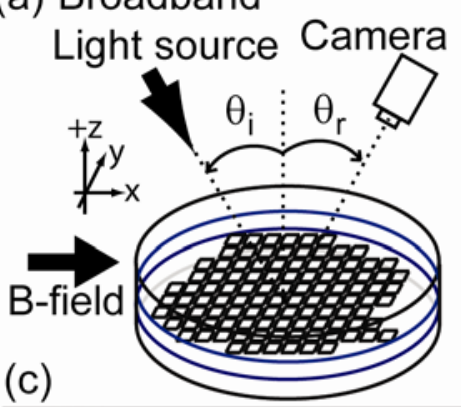
(b)

Figure 4. Optical micrographs of the tiles oriented under different directions $(\theta)$ of magnetic field. The length and width of the tiles were both $2 \mathrm{~mm}$.

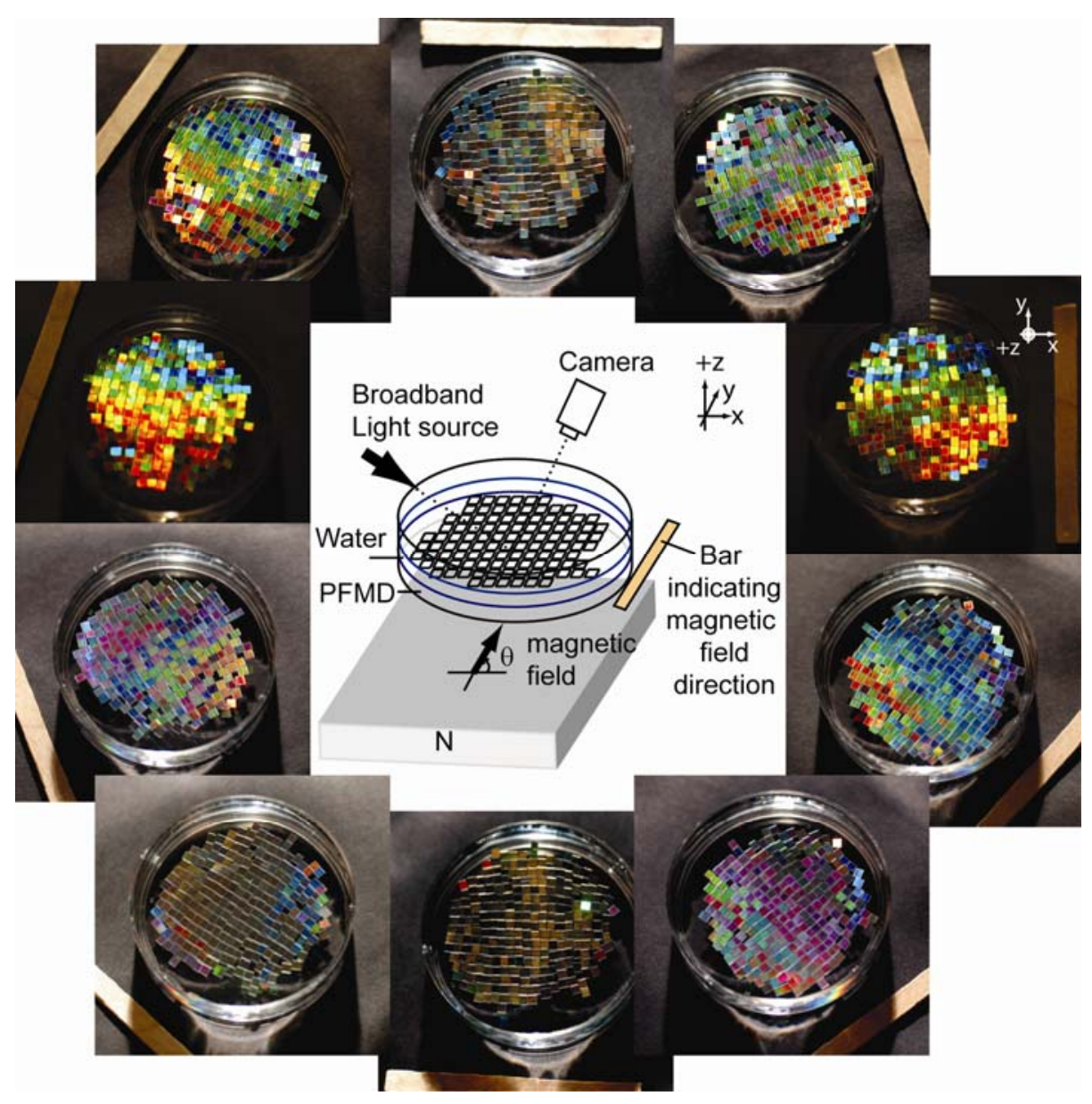


Figure 5. Packing structures of the tiles differed according to the pattern of magnetic field applied. (a) shows the tiles in the absence of magnetic fields. The length and width of each tile were both $2 \mathrm{~mm}$.

(a) (i)



(b) (i)



(c) (i)



(d) (i)

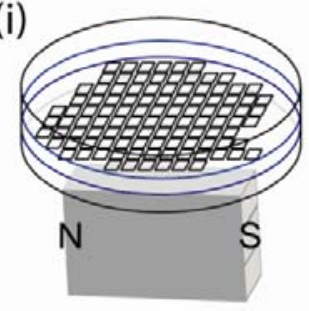

(e) (i)

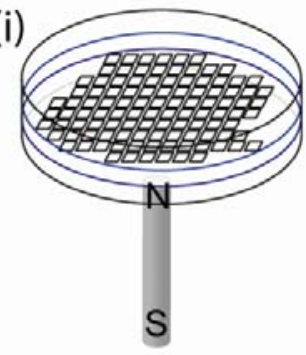

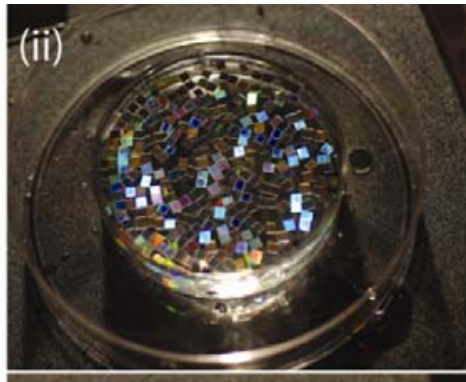

Figure 6. (a) Scheme showing the transfer of the tiles from the liquid-liquid interface to a solid substrate. (b) shows assembled circular tiles at the liquid-liquid interface. The diameter of the tiles was $2 \mathrm{~mm}$. (c) and (d) show the bottom of the tiles after agarose gelled. Some tiles were displaced and lost when the gel was transferred between different surfaces (for photography) by hand. These displacements can be avoided by transferring the gel directly onto PDMS. (e) and (f) are photographs of tiles transferred onto a film of PDMS that was curved, and wrapped around a wrist respectively.

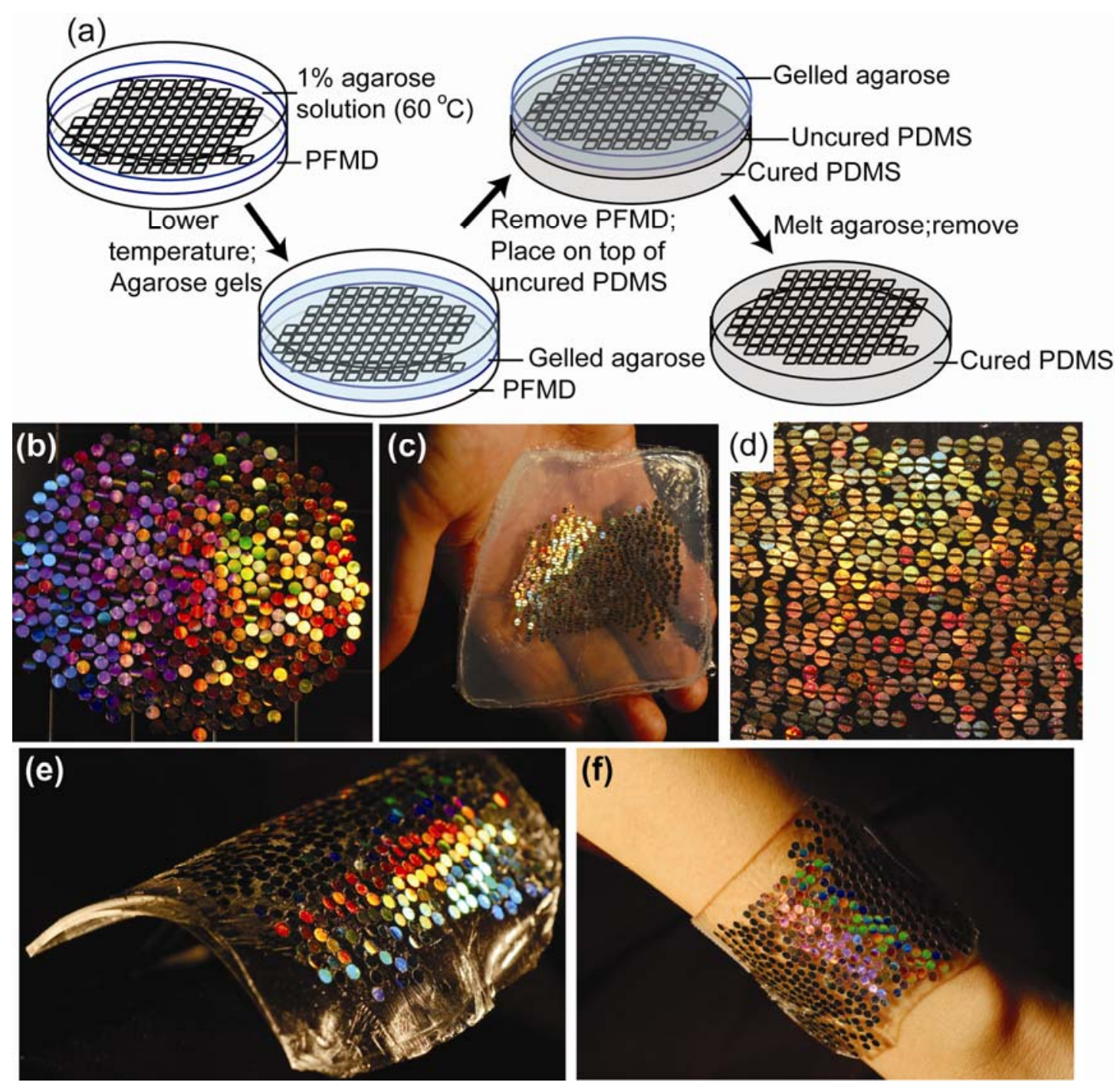

